\title{
Graphene quantum dots: From efficient preparation to safe renal excretion
}

Caroline Hadad ${ }^{1,2, \S}$, José Miguel González-Domínguez ${ }^{1,3, \S}$, Silvia Armelloni ${ }^{4, \pi}$, Deborah Mattinzoli ${ }^{4, \pi}$, Masami Ikehata $^{4}$, Akcan Istif ${ }^{1}$, Adrian Ostric ${ }^{1}$, Francesco Cellesi ${ }^{5}$, Carlo Maria Alfieri ${ }^{4,6,7}$, Piergiorgio Messa ${ }^{4,6,7}$, Belén Ballesteros $^{8}$, and Tatiana Da $\operatorname{Ros}^{1}(\bowtie)$

${ }^{1}$ Centre of Excellence for Nanostructured Materials (CENMAT), INSTM, Department of Chemical and Pharmaceutical Sciences, University of Trieste, Via L. Giorgieri 1, 34127 Trieste, Italy

${ }^{2}$ Current address: Laboratoire LG2A - CNRS UMR 7378, Université de Picardie Jules Verne, 33 rue Saint Leu - UFR des Sciences, 80039 Amiens, France

${ }^{3}$ Instituto de Carboquímica (CSIC), C/Miguel Luesma Castán 4, E-50018 Zaragoza, Spain

${ }^{4}$ Renal Research Laboratory-Fondazione IRCCS Ca' Granda Ospedale Maggiore Policlinico, via Pace 9, 20122 Milan, Italy

${ }^{5}$ Dipartimento di Chimica, Materiali ed Ingegneria Chimica "G. Natta", Politecnico di Milano, Via Mancinelli 7, 20131 Milan, Italy

${ }^{6}$ Unit of Nephrology, Dialysis and Renal Transplant Fondazione IRCCS Ca' Granda Ospedale Maggiore Policlinico, via della commenda 4, 20122 Milan, Italy

${ }^{7}$ Department of Clinical Sciences and Community Health, University of Milan, 20122 Milan, Italy

${ }^{8}$ Catalan Institute of Nanoscience and Nanotechnology (ICN2), CSIC and the Barcelona Institute of Science and Technology, Campus UAB, Bellaterra, 08193 Barcelona, Spain

${ }^{\S}$ Caroline Hadad and José Miguel González-Dominguez contributed equally to this work.

"Silvia Armelloni and Deborah Mattinzoli contributed equally to this work.

(C) The Author(s) 2020

Received: 23 June 2020 / Revised: 3 September 2020 / Accepted: 6 September 2020

\section{ABSTRACT}

Carbon nanomaterials offer excellent prospects as therapeutic agents, and among them, graphene quantum dots (GQDs) have gained considerable interest thanks to their aqueous solubility and intrinsic fluorescence, which enable their possible use in theranostic approaches, if their biocompatibility and favorable pharmacokinetic are confirmed. We prepared ultra-small GQDs using an alternative, reproducible, top-down synthesis starting from graphene oxide with a nearly $100 \%$ conversion. The materials were tested to assess their safety, demonstrating good biocompatibility and ability in passing the ultrafiltration barrier using an in vitro model. This leads to renal excretion without affecting the kidneys. Moreover, we studied the GQDs in vivo biodistribution confirming their efficient renal clearance, and we demonstrated that the internalization mechanism into podocytes is caveolae-mediated. Therefore, considering the reported characteristics, it appears possible to vehiculate compounds to kidneys by means of GQDs, overcoming problems related to lysosomal degradation.

\section{KEYWORDS}

graphene quantum dots, podocytes, biodistribution, uptake pathway, renal clearance, nanocarrier

\section{Introduction}

Since 2004, the landmark study by Geim and Novoselov drew the attention into graphene, that presents undisputed potential in many areas, particularly in optoelectronics, due to its unique electronic structure and overall physical properties [1]. Its oxidized derivative, graphene oxide (GO) is also largely popular for its high hydrophilicity, easy handling and affordable production. Also in this case there is an interesting niche of applications, as electrochemistry [2] and biotechnology [3] and from the thorough study of GO and related derivatives, novel discoveries emerged. In 2008 Sun et al. [4] realized on the potential of nanometric-sized flakes of GO for biomedicine, as these possessed luminescent properties coupled to multifunctionalization possibilities for both cellular imaging and anticancer drug delivery in specific cell lines. This back- then-called "nano graphene oxide" showed unprecedented biocompatibility and practical value, being the first application example of what is known today as graphene quantum dots (GQDs).

GQDs consist of tiny crystalline zero-dimensional pieces of graphene, generally oxidized in a certain degree, receiving their designation from the analogy with traditional semiconductor quantum dots (e.g. CdSe). Their small size is enough to cause quantum phenomena by exciton confinement and edge effects, resulting in photoluminescent (PL) properties. Graphene exhibits a zero electronic bandgap, but a fragment of graphene (technically of any size if small enough) will display a non-zero bandgap leading to luminescence upon excitation [2]. This bandgap can be tailored through the GQDs geometry, size and chemical composition of their edges [5], giving rise to a wide range of possible emissions. Unlike the classical semiconductor 
quantum dots, GQDs are intrinsically water-soluble and rather easy to handle at a chemical level, since they can be treated almost as molecules, and not as unstable/metastable colloids, opening many venues for their study.

The rapid rise of these nanostructures in recent years all over the world has generated different nanostructures termed as GQDs or GO quantum dots (GOQDs) with slight variations in chemical composition and shape, mostly depending on their synthesis route and nature of the carbon precursor. This diversity would need a systematization of nomenclature according to the presence of the graphene lattice (to properly distinguish GQDs from carbon dots), the lateral size, the thickness and degree of oxidation in a similar way as done for graphene [6], in order to delimit the structural and compositional conditions in which the quantum confinement stands. However, until that happens, scientists will tend to use the terms GQDs and GOQDs indistinctly. With a lateral size ranging from few nanometers to $100 \mathrm{~nm}$ (typically up to $20 \mathrm{~nm}$ ), and in the form of one or few layers, all less than $10 \mathrm{~nm}$, these carbon nanoforms can be obtained via top-down or bottom-up approaches [6-8]. The top-down methodology seems to be the most followed for the production of GQDs, as it usually grants larger-scale outputs besides being highly versatile and readily accessible $[9,10]$. The choice of the carbon precursor determines many features of the eventual nanostructures, such as its shape [9], heteroatom doping [11], and photophysical properties [12], hence a huge diversity of feedstocks have been reported in literature for this purpose, including biomass wastes, mineral carbons, carbon nanostructures, synthetic polymers and biological entities [7]. As regards to their PL properties, GQDs display a typically broad emission dependent on the excitation wavelength, and these can be easily tuned, apart from the GQDs own features, throughout $\mathrm{pH}$, dispersion medium or even upon postsynthesis surface modification [12].

GQDs possess an unparalleled biocompatibility, chiefly stemming from their high water-solubility, reason why these nanostructures are intensively applied in biological or biomedical studies, showing exceedingly higher biocompatibilities than semiconductor quantum dots, even in the absence of chemical derivatization [13]. Some works dealing with bioimaging, biosensing, and disease treatments are reported in literature, suggesting that GQDs have excellent biocompatibility, with relatively low in vitro and in vivo toxicity [14]. Overall, GQDs seem much less toxic than GO as reported in many studies. In this context, Chong et al. described the synthesis of GQDs by a graphite powder modified cutting method and found that, unlike GO, which tends to form aggregates, no obvious in vitro and in vivo toxicity was found, even under a multi-dosing situation [15].

Not many papers reported about the biodistribution of GQDs and one of the first was by Chong and co-workers [15]. Other experiments on GQDs obtained by an electrochemical method and presenting dimensions in the range of 160-280 nm, were performed, in which the GQDs were directly radiolabeled with ${ }^{99 \mathrm{~m}} \mathrm{Tc}$ and used for in vivo studies on mice and for in vitro mutagenicity tests. While from the in vitro experiments some mutagenic activity emerged with changes from A:T to $\mathrm{G}: \mathrm{C}$, the in vivo experiments resulted in good biocompatibility and an equal biodistribution in healthy mice organs. Instead, the case of endometriosis model mice, a strong increase in the uptake by kidneys and of the renal clearance was reported [16].

To exert the in vivo safety, studies were also performed on zebrafish embryos, which properly grew despite the GQDs accumulation in the digestive tract, tracked using the GQDs photoluminescence [17]. Analogously, reduced GOQDs
(rGOQDs) were tested and a dose-dependent variation of the animal development was reported [18]. Analyses on zebrafish larvae at different concentrations $(12.5-200 \mu \mathrm{g} / \mathrm{mL})$ resulted in embryo malformations with the higher concentrations treatment, while the lower resulted to be safe [19]. To evaluate the biodistribution also radioactive tracking was used. Carbon materials obtained by the pyrolysis of citric acid were functionalized using $\mathrm{Na}^{131} \mathrm{I}$. Also in this case, despite the different source and decoration of the materials, the fate was the same, with higher concentrations in liver and kidney, suggesting renal excretion. Moreover, in tumor bearing mice, the concentration in the affected tissue was quite good [20].

Despite all these encouraging results, the toxicity of GQDs is an important subject, which must be further addressed, since there is still a lack of knowledge for many unexplored cell lines and in vivo models and, moreover, the studied materials often differ for chemical and physical characteristics. In particular, it would be of a great interest to confirm their biodistribution in vivo in more models and to unravel the exact excretion mechanisms in view of a future application of GDQs in bioimaging or nanomedicine.

The present work describes the reproducible top-down synthesis of GQDs starting from GO with a nearly $100 \%$ conversion of the starting material. Cytotoxicity of the obtained GQDs was then assessed in vitro on human renal cells, and in mice. Indeed, vascular endothelial cells, glomerular basement membrane (GBM) and podocytes represent the main components of glomerular filtration barrier, whose alteration compromise renal function and results in proteinuria, a sign of glomerular disease [21]. For this reason, these cells constitute an appealing target for nanoparticles (NPs) drug delivery. A model of glomerular filtration barrier, composed of endothelial cells, collagen IV, a protein of GBM, and podocytes, were used to examine GQDs passage [22]. Moreover, the evaluation of cytoskeletal proteins distribution was assessed in podocytes after NPs addition; F-actin represents an important marker to assess the maintenance of the correct cellular morphology and the functional integrity $[23,24]$ and was used to verify GQDs biocompatibility.

Podocytes are highly differentiated cells characterized by a large cytoplasm with primary and secondary processes, protruding and interconnecting [25]. As known, size and charge of biomolecules determine NPs repulsion or absorption by the barrier [26]. Only NPs below $80 \mathrm{~nm}$ pass the endothelial fenestration and lower than $32 \mathrm{~nm}$ the ultrafiltration barrier of podocytes, consisting of a bridge between the secondary processes. The impairment of these components may occur during primary and acquired nephropathies and the delivery of the drugs directly on damage cells, may constitute the goal of GQDs use in human therapy.

\section{Experimental}

\subsection{Material and methods}

\subsubsection{Reagents, materials and instruments}

\section{Chemical part}

Mineral acids, used at higher commercially available concentration, were from Sigma-Aldrich (Milan, Italy) or Alfa Aesar (Thermo Fisher, Milan, Italy). Dialysis sacks were from Sigma Aldrich D6066. High purity graphite powder was from Carbon Bay (grade SP1, batch \#04100, lot \#011705). Milli-Q water (resistivity of $18 \mathrm{M} \Omega \cdot \mathrm{cm}$ ), obtained from a Millipore system, was used in all experiments and cleaning steps. thermogravimetric analysis 
(TGA) was recorded on a TGA Q500 (TA instruments), under $\mathrm{N}_{2}$ flow, by equilibrating at $100{ }^{\circ} \mathrm{C}$, and following with a ramp at $10^{\circ} \mathrm{C} / \mathrm{min}$ up to $800^{\circ} \mathrm{C}$ (approximately $1 \mathrm{mg}$ of each compound). High-resolution transmission electron microscopy (HR-TEM) and HAADF-STEM images and energy-dispersive $\mathrm{X}$-ray spectroscopy (EDX) spectra were acquired using a FEI Tecnai G2 F20 microscope operated at $200 \mathrm{kV}$ coupled to an EDAX super ultra-thin window (SUTW) X-ray detection system. Samples were prepared by drop casting from the GQDs aqueous solution onto a TEM grid (300 mesh, ultrathin carbon on holey carbon coated copper grid). Infrared spectra were obtained by using a Fourier-transform infrared (FT-IR) spectrometer (Perkin-Elmer 2000) through pellets with spectroscopic grade $\mathrm{KBr}$. The ultraviolet-visible-near-infrared (UV-Vis-NIR) spectroscopy measurements were carried out with a Varian Cary 5000 spectrophotometer by using $1 \mathrm{~cm}$ path length polystyrene cuvettes. Fluorescence emission spectra have been recorded on a Varian Cary Eclipse Spectrophotometer using $10 \mathrm{~mm}$ path length Hellma Analytics 117.100F-QS quartz cuvettes. For atomic force microscopy (AFM) visualization, the samples were prepared by drop-casting the GQDs aqueous solution onto $\mathrm{SiO}_{2} / \mathrm{Si}$ wafer substrates and dried under vacuum. Measurements were performed with a Nanoscope V (Digital Instruments Metrology Group, model MMAFMLN) in tapping mode in air at room temperature, using an n-type silicon $\mu$ mash ${ }^{\circledR}$ SPM probe (HQ:NSC15/AL BS) with tip height $12-18 \mu \mathrm{m}$, cone angle $<40^{\circ}$ (Resonant frequency $325 \mathrm{kHz}$, force constant of $\sim 40 \mathrm{~N} / \mathrm{m}$ ). The collected images were then analyzed with WsXm 4.0 software (Nanotec Electronica S. L.), and Gwyddion 2.39. Dispersions of GO in sulfonitric mixture were sonicated in a temperature-controlled bath sonicator (Elmasonic S, $37 \mathrm{kHz}$ ).

\section{Biological part}

Hemangioendothelioma Endothelial Cells (EOMA) (ATCC ${ }^{\circledR}$ CRL-2586 ${ }^{\mathrm{TM}}$ ) were from LGC Standards S.r.l. (Sesto San Giovanni, Milan, Italy). Podocytes SV1 cell line, from transgenic $\mathrm{H}-2 \mathrm{~Kb}$-tsA58 mice, were from Cell Line Service (Eppelheim Germany). 96-well plates used to grow cells were from Corning (Milan, Italy). All reagents used for culture maintenance were from Sigma-Aldrich as well as Trypan blue staining phalloidin 488 probe and 4',6-diamidino-2-phenylindole (DAPI). The LDH-Cytotoxicity Colorimetric Assay Kit was purchased by Bio Invision Incorporated Vinci-Biochem (Firenze, Italy), the tetramethylrhodamine-albumin bovine serum by Invitrogen, while paraffin was from Histo-line laboratories (Milan, Italy), optimal cutting temperature compound by Sakura Finetek Europe B.V. (Netherlands), and Carazzi's Hematoxylin-Eosin from Bio-Optica (Milan, Italy). Polyethylene terephthalate (PET) membrane $1 \mu \mathrm{m}$ porous (Millicell hanging cell culture inserts) was from Millipore (Milan, Italy). Mice C57BL/6N were from Charles River Laboratories (Calco, Lecco, Italy). Coverslips were supplied by Thermanox, Nunc, VWR Int. (Milan, Italy).

Absorbance was obtained by Xenius SAFAS spectrophotometer (Safas, Monaco). Immunofluorescence images were acquired by Zeiss AxioObserver microscope equipped with high-resolution digital videocamera (AxioCam, Zeiss) and Apotome system for structured illumination and recorded by AxioVision software 4.8. Histological slices were observed by Zeiss Axioscope 40 microscope equipped with AxioCam MRc5 digital camera. Histological images were then acquired by Zeiss AxioObserver microscope equipped with high resolution digital videocamera (AxioCam, Zeiss) and Apotome system for structured illumination and recorded by AxioVision software 4.8. Fluorescence spectroscopy measurements were performed using a Xenius SAFAS spectrophotometer.

\subsection{Procedures}

\subsubsection{Synthesis of graphite oxide (GO)}

GO was obtained according to the modified Hummer's method. $3.11 \mathrm{~g}$ of graphite powder and $1.54 \mathrm{~g}$ of sodium nitrate were added into $70 \mathrm{~mL}$ concentrated $\mathrm{H}_{2} \mathrm{SO}_{4}$ at $0{ }^{\circ} \mathrm{C}$ and stirred for 30 minutes. After this time, $\mathrm{KMnO}_{4}(9.06 \mathrm{~g})$ was gradually added to the mixture under continuous stirring in ice-bath for 2-3 minutes. Then, the mixture was heated at $35{ }^{\circ} \mathrm{C}$ for 30 minutes and $138 \mathrm{~mL}$ of water were added. The temperature of the mixture was increased until $100{ }^{\circ} \mathrm{C}$ and maintained for 15 minutes. The obtained brown solution was diluted into $420 \mathrm{~mL}$ of water under stirring and the reaction was quenched after addition of $3 \mathrm{~mL}$ of $\mathrm{H}_{2} \mathrm{O}_{2}$. Then, the stirring was switched-off, the graphite oxide (brown suspension) settled to the bottom overnight and the orange supernatant was decanted off. $200 \mathrm{~mL}$ of water were added to the graphite oxide and the mixture was stirred for 5 minutes. After $1 \mathrm{~h}$ of settlement, the graphite oxide deposited in the bottom and the supernatant was removed. This operation was repeated 3 times. $100 \mathrm{~mL}$ of water were added to the brown deposit and stirred for 10 minutes. The mixture was then transferred into dialysis sacks against Milli-Q water until pH 4-5 and then lyophilized to obtain $2.85 \mathrm{~g}$ of GO.

\subsubsection{GQDs preparation}

In a typical experiment, 6 sealed glass vials of $20 \mathrm{~mL}$ containing $10 \mathrm{mg}$ of GO and $2.5 \mathrm{~mL}$ of acidic mixture (concentrated $\mathrm{H}_{2} \mathrm{SO}_{4} / \mathrm{HNO}_{3}$ mixture, $3: 1$ ) were sonicated for $24 \mathrm{~h}$ at $80{ }^{\circ} \mathrm{C}$ using a sonic bath (if necessary, vial caps were replaced every $8 \mathrm{~h}$ of sonication). After this time elapsed, vials were removed from the sonic bath, allowed to cool down to room temperature and the dark brown liquids contained in each vial were carefully diluted with Milli-Q water until the top of the vial (8-fold dilution) and hand-shaken for a few seconds. After settling down at room temperature overnight, hand shaking was again performed and the orange solutions were gathered together and transferred into dialysis sacks. The dialysis against Milli-Q water took place with frequent water replacements, until the $\mathrm{pH}$ of the outer waters became neutral. It is important to mention that a careful and thorough tracing of the dialysis process was undertaken, and no fluorescence was ever noticed in the dialysis waters, neither carbonaceous solids upon filtrating them, meaning that GQDs fully remained inside the dialysis sacks. Once neutralized, the resulting yellow solution of GQDs $(0.22 \pm 0.04 \mathrm{mg} / \mathrm{mL})$ was taken out from the sacks, protected from the light and stored at $3-4{ }^{\circ} \mathrm{C}$ until further characterization or use. By freeze-drying this solution, a spongy and fluffy light solid was obtained and used for TGA and IR analyses.

\subsubsection{Mouse podocytes and hemangioendothelioma endothelial cells line culture}

EOMA were maintained in incubator at $37^{\circ} \mathrm{C}$ at $5 \% \mathrm{CO}_{2}$, in Minimum Essential Medium Eagle (EMEM) supplemented with $10 \%$ fetal bovine serum and $1 \%$ penicillin/streptomycin, according to manufacturer's protocol. Podocytes were previously expanded at $33^{\circ} \mathrm{C}$ in DMEM:F12 medium supplemented with $10 \% \mathrm{FCS}, 100 \mathrm{U} / \mathrm{mL}$ penicillin, $100 \mu \mathrm{g} / \mathrm{mL}$ streptomycin, $2 \mathrm{mM}$ L-glutamine and $20 \mathrm{U} / \mathrm{mL}$ recombinant mouse $\gamma$ interferon. Subsequently the podocytes were grown in culture flasks precoated with collagen type IV in a complete medium, without gamma interferon and thermoshifted to $37^{\circ} \mathrm{C}$ for 7 days 
of differentiation. All experiments were then conducted on differentiated podocytes.

\subsubsection{Cytotoxicity assessment by LDH assays}

For the cytotoxicity assessments, podocytes and EOMA cells were seeded at $104 \mathrm{cell} / \mathrm{s} / \mathrm{cm}^{2}$ in triplicate into 96-well plates in a basal medium till $60 \%$ of confluence. Lactate dehydrogenase assays (LDH) were then performed on both SV1 podocytes and EOMA cells according to manufacturer's instructions. A concentration ranges ( 10 to $100 \mu \mathrm{g} / \mathrm{mL}$ ) of GO and GQDs, for 24 hours at $37^{\circ} \mathrm{C}$ have been used. As positive control, $10 \mu \mathrm{L}$ of lysis solution was added for the same time. At the end of incubation, the plate was gently shacked and centrifuged at $600 \mathrm{~g}$ for 10 minutes. Supernatant $(10 \mu \mathrm{L})$ was then transferred into new 96 well-plate and the reaction mix was added to each well and incubated at room temperature for 1 hour. After adding stop solution, the absorbance was measured at $450 \mathrm{~nm}$ and the value of background signal at $680 \mathrm{~nm}$ was subtracted.

\subsubsection{Assessment of GQDs permeability through glomerular filtration barrier}

The use of a three-dimensional model of glomerular filtration barrier allowed assessing the GQDs passage through the artificial barrier. As previously described by some of us [22], after coating on both sides PET membranes insert with Type IV collagen, EOMA cells were seeded on the lower side of the membrane and cultured for 1 week in the presence of $5 \mathrm{ng} / \mathrm{mL}$ of VEGF. Once cells attached, podocytes were seeded on the opposite side of the membrane and incubated in their own complete medium for differentiation. Each insert was placed in a 24-well plate with the EOMA side facing the bottom of the well. GQDs $30 \mu \mathrm{g} / \mathrm{mL}$ were added to EOMA side in each well. At different time point (30 minutes, 1, 2, 4 and 24 hours) medium on the side of podocytes was collected and optical density was measured by spectrophotometer at $550 \mathrm{~nm}$ of excitation and $570 \mathrm{~nm}$ of emission respect to a GQDs concentration curve. The time dependent curve obtained was then compared to the tetramethylrhodamine-albumin bovine serum (TRITC-BSA) curve detected at the same wavelength. The BSA permeability, used as control, was obtained adding $40 \mathrm{mg} / \mathrm{mL}$ of TRITC-BSA to the cells instead of GQDs. The kinetic and the amount of GQDs and BSA that permeated through the glomerular-like artificial filtration barrier were compared.

\subsubsection{Evaluation of GQDs cellular uptake}

Podocytes $\left(20,000\right.$ cells $\left./ \mathrm{cm}^{2}\right)$ were grown on coverslips at $50 \%$ of confluences, the cells were then treated with $30 \mu \mathrm{g} / \mathrm{mL}$ of GQDs and observed after 30, 60 and 90 minutes. In the aim to deepen the uptake pathway, podocytes were pre-incubated for 30 minutes with different endocytosis inhibitors at concentrations previously tested from some of us [27]: filipin III $1 \mu \mathrm{g} / \mathrm{mL}$, genistein $200 \mu \mathrm{M}$, wortmannin $100 \mathrm{nM}$, chlorpromazine hydrochloride $10 \mu \mathrm{g} / \mathrm{mL}$, sodium azide $10 \mathrm{mM}$. Three different experimental conditions were used: control with basal medium, inhibitors in basal medium and inhibitors followed by of GQDs. Trypan blue staining vitality test was performed after the incubation with the selected inhibitors to reconfirm the cell death below $50 \%$, after 150 minute of exposure. The amount of quantum dots uptake was assessed through the quantification of the GQDs fluorescence on 5 fields.

To evaluate a possible cytoskeleton damage after treatment, the cells were fixed with $4 \%$ PFA for 10 minutes at each time point. F-actin cytoskeletal morphology of podocytes was then investigated by a direct immunofluorescence with phalloidin
488 probe and the nuclei were stained by DAPI.

\subsubsection{Animal model and ethics statement}

All procedures involving animals were performed in accordance with the European legislation (European Communities Council Directive of 24 November 1986, 86/609/ EEC) and complied with the European Communities Parliament and Council Directive of 22 September 2010 (2010/63/EU) and with the Italian (D.L.vo n. 26/2014) and the Protocol N. 07/13 approved by the Milan University Institutional Care and Ethical Treatment Committee. Eighteen mice C57BL/6N of 3 months of age were housed in plastic cages ( $5 \mathrm{mice} / \mathrm{cage}$ ), according to the current ethical and legislative rules Directive 2010/63/EU for animal experiments. They were maintained under controlled conditions of humidity, temperature and 12 hour light/dark cycles; all animals allowed free access to sterile pelleted food and water ad libitum.

\subsubsection{Evaluation of in vivo GQDs biodistribution}

Animals were then randomly divided in groups of three and intravenous injections (IV) of $200 \mu \mathrm{L}$ of GQDs solution at the final concentration respectively of 10,25 and $50 \mu \mathrm{g} / \mathrm{mL}$ were performed. For each group three mice were injected with $200 \mu \mathrm{L}$ of vehicle as control. After $24 \mathrm{~h}$ mice have been sacrificed after anesthesia induced by intraperitoneal injection of $370 \mathrm{mg} / \mathrm{kg}$ of chloral hydrate and brain, liver, kidney, lung, spleen, stomach, colon, and bladder were excised and urine collected for further investigation.

\subsubsection{Histological analyses}

Brain, liver, kidney, spleen, lung, stomach, colon, and bladder were embedded in paraffin and optimal cutting temperature compound. Paraffin sections ( $5 \mu \mathrm{m}$ thick) were de-waxed in xylene and rehydrated in gradient alcohol for 5 minutes. Carazzi's Hematoxylin-Eosin staining were performed according to standard protocol. Sections were then observed under microscope. 5 sections were prepared for subsequent analysis. Frozen tissue ( $4 \mu \mathrm{m}$ thick) were fixed in PFA $4 \%$ for 10 seconds and stained with DAPI to visualize the nuclei, images were acquired for tissue GQDs distribution determination.

\subsubsection{GQDs quantification in the urine}

Upon injection, each mouse was housed in a metabolic cage to collect 24 hour urine. Controls and treated mice urines were diluted with deionized water to a final volume of $1 \mathrm{~mL}$ and $100 \mu \mathrm{L}$ were used to measure the emission by fluorescence spectroscopy.

\section{Results}

\subsection{Synthesis and characterization of GQDs}

Graphite oxide was first obtained from graphite powder using the so-called Hummers method [28]. This method is by far the one of the most widely used for graphite oxidation, which involves the use of concentrated acids and the presence of a strong oxidizing agent. After purification steps mainly based on dialysis against Milli- $\mathrm{Q}$ water, the resulting material was used as a source for the synthesis of GQDs. Therefore, GQDs in aqueous solution were obtained after sonication of GO in a mixture of concentrated acids (i.e. sulfonitric mixture) at $80^{\circ} \mathrm{C}$ for $24 \mathrm{~h}$ (Fig. 1). After dilution, the presence of water helps the stabilization of the GQDs in acid environment, being the excess of acids subsequently eliminated by dialysis until reaching neutral $\mathrm{pH}$. The concentration of GQDs in water was determined after lyophilization of different aliquots. By 

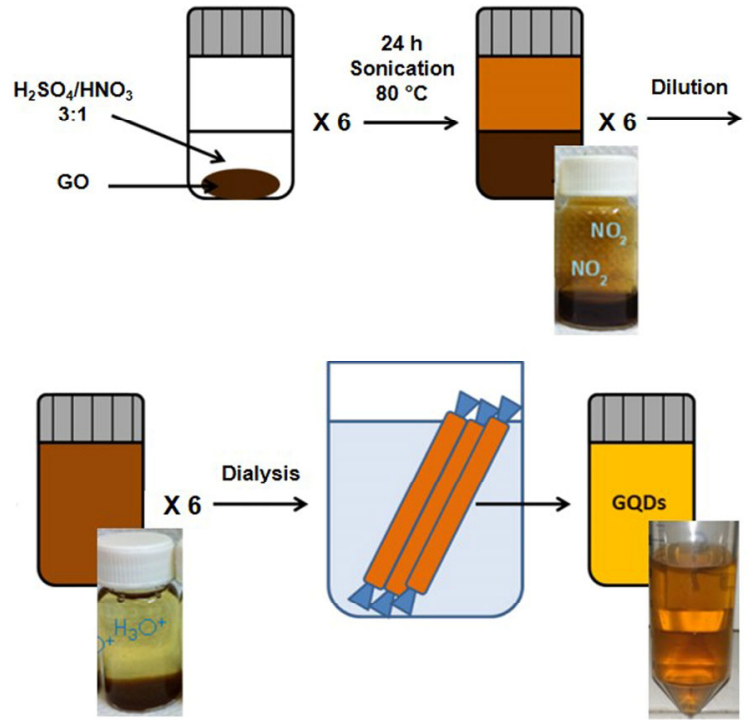

Figure 1 Schematic representation of the GQDs synthesis. Procedure process to prepare GQDs.

freeze-drying of the obtained solution, the concentration of GQDs was determined as $0.22 \pm 0.04 \mathrm{mg} / \mathrm{mL}$ and their production was virtually of $100 \%$, since no traces of GO were found anywhere along the process, as it was checked by $\mathrm{UV}-\mathrm{Vis}$ and atomic force microscopy (AFM) analyses of the elutes, and the described methodology did not involve any size-separation step.

Both GO and GQDs were characterized using TGA, Raman and IR spectroscopies, TEM, AFM and, in the case of the GQDs, EDX. TGA performed under nitrogen atmosphere (Fig. 2(a)) showed that GO and GQDs are thermally unstable and started to lose mass upon heating above $100{ }^{\circ} \mathrm{C}$, with a significant drop in mass at $212^{\circ} \mathrm{C}$ (around $30 \%$ of the total mass) for GO and at 164 and $202{ }^{\circ} \mathrm{C}$ for GQDs (around $50 \%$ of the total mass) corresponding to the thermal desorption of the labile oxygen-containing groups (e.g. carboxylic, hydroxyl or epoxide groups). The oxidation of graphite to GO was also confirmed by FT-IR spectroscopy (Fig. 2(b)). The spectra show the characteristic bands at 3,400,1,731 and $1,647 \mathrm{~cm}^{-1}$ assigned to the $\mathrm{O}-\mathrm{H}$ group stretching vibrations and to $\mathrm{C}=\mathrm{O}$ stretching of carboxylic and/or carbonyl moiety functional groups respectively but also two peaks at about 1,238 and $1,072 \mathrm{~cm}^{-1}$ corresponding to the $\mathrm{C}-\mathrm{O}$ stretching vibrations.

The Raman spectra (Fig. 2(c)) showed the main features of graphitic carbon-based materials with the $G$ and $D$ peaks at around 1,609 and $1,349 \mathrm{~cm}^{-1}$, corresponding to $\mathrm{sp}^{2}$ vibrations and to the disorder-induced by the defects on the $\mathrm{sp}^{2}$ hybridized hexagonal sheet of the carbon, respectively.

The morphology of GO and GQDs was investigated through microscopic observation. In the case of GO, large single- or few-layer sheets were observed in TEM, reaching several micrometers in lateral size (see Fig. S1 in the Electronic Supplementary Material (ESM)). These large sheets of graphene oxide were cut during the synthesis of GQDs ending up in an average diameter of $2.9 \pm 0.8 \mathrm{~nm}$ determined by HR-TEM (Fig. 3). Preliminary DLS measurements of the lyophilized material dispersed in water at a concentration of $100 \mu \mathrm{g} / \mathrm{mL}$ resulted in a hydrodynamic radius of $26.2 \pm 1 \mathrm{~nm}$ (Fig. S4 in the ESM). From HR-TEM, interplanar spacings of $0.21 \mathrm{~nm}$, corresponding to the (100) plane in GQDs, were observed in some of the particles (Fig. 3(b), inset). EDX analysis on the GQDs showed the characteristic peaks for carbon and oxygen, as expected, and also a small amount of sulfur, which might
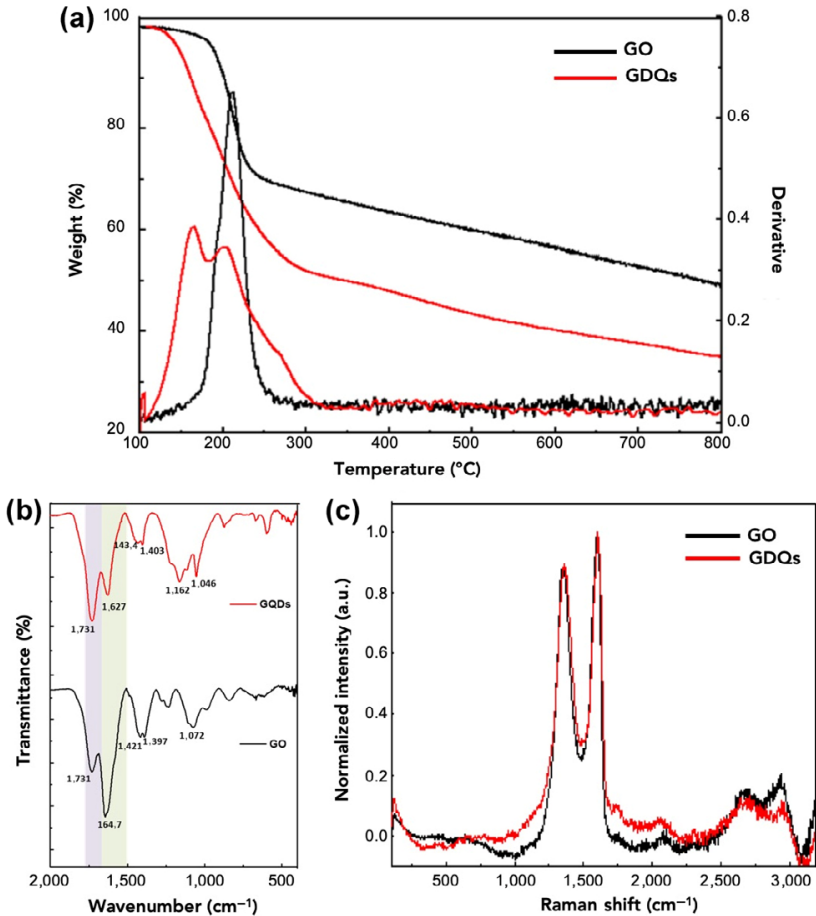

Figure 2 Characterization of GO and GQDs. (a) TGA analyses of GO and GQDs under nitrogen atmosphere. (b) FT-IR spectra of GO and GQDs, KBr pellets. (c) Raman spectra of GO and GQDs, $\lambda=532 \mathrm{~nm}$. GO: black line; GQDs: red line.
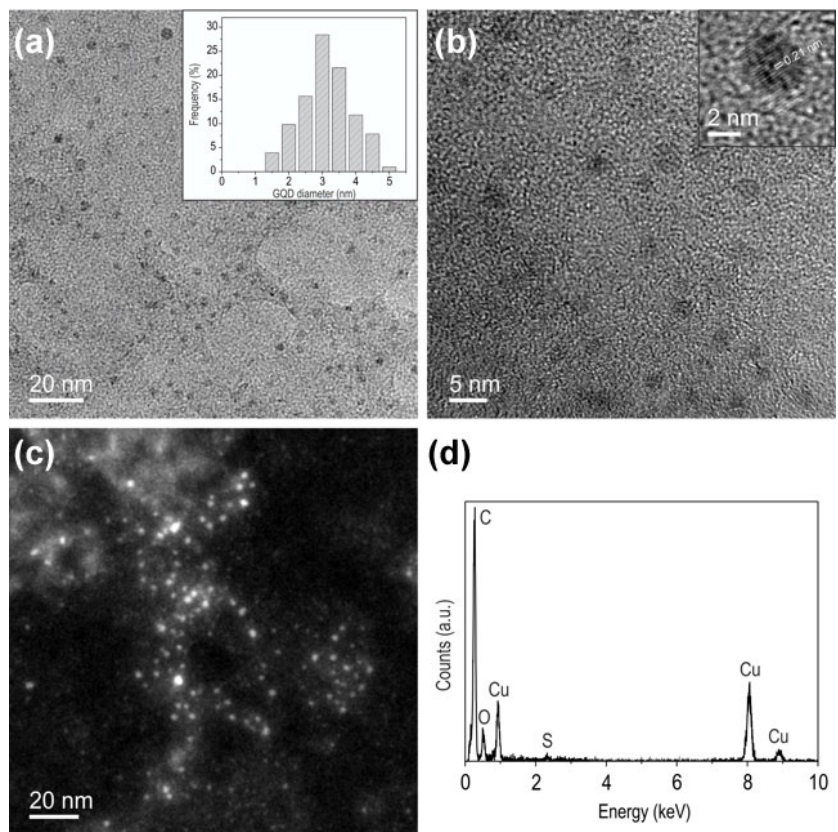

(d)

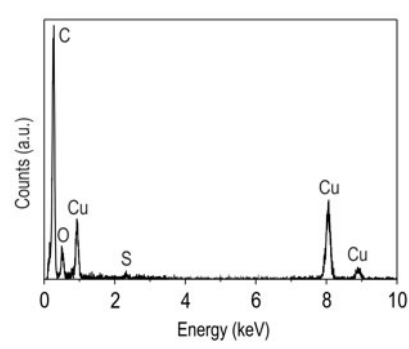

Figure 3 HR-TEM images of GQDs. ((a) and (b)) HR-TEM images of GQDs, size distribution histogram derived from HR-TEM images (inset in (a)) and image of the crystalline graphene structure, with $0.21 \mathrm{~nm}$ interplanar spacing corresponding to the (100) planes (inset in b)). (c) High-angle annular dark-field (HAADF)-STEM image of GQDs. (d) EDX spectrum of GQDs. Scale bars: ((a) and (c)) $=20 \mathrm{~nm},(\mathrm{~b})=5 \mathrm{~nm}$. The distribution histogram is based on analysis of 102 GQDs.

arise from the $\mathrm{H}_{2} \mathrm{SO}_{4}$ used for their preparation. The copper signal comes from the TEM grid.

The AFM characterization was used to ascertain the number of layers of which GQDs were made of, as appreciable from Fig. 4 with different magnifications of a deposited sample of GQDs, revealing that their height ranged between 1 and $2 \mathrm{~nm}$ (mostly below $1.5 \mathrm{~nm}$ ). 

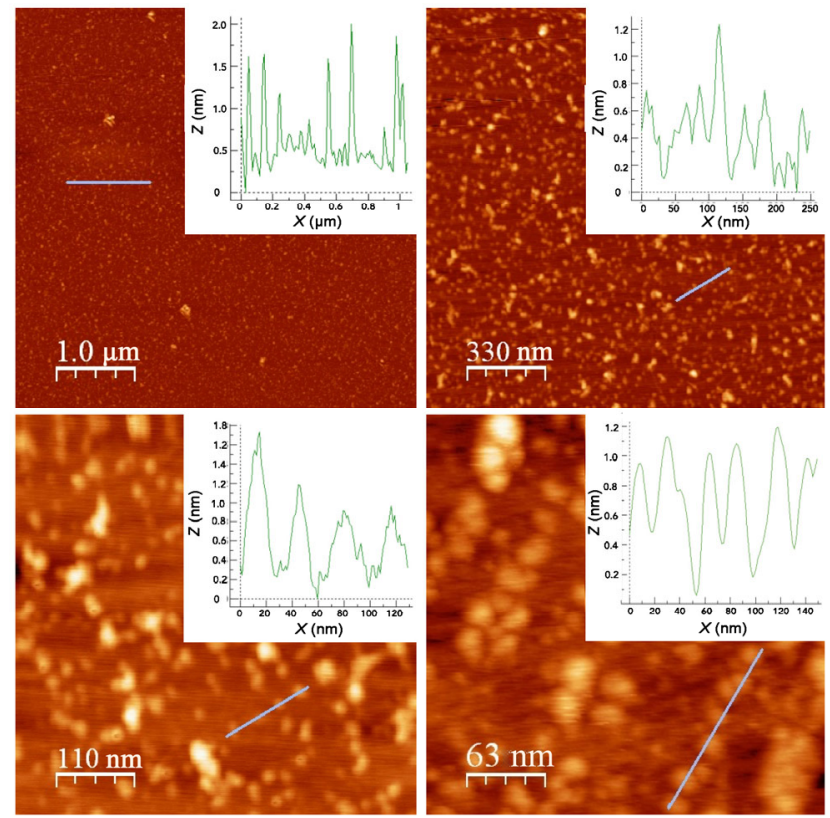

Figure 4 AFM images of GQDs. Different AFM images of GQDs with their respective height profiles along the drawn line in each image.

The typical fluorescence emission from the GQDs (Fig. S2 in the ESM, in blue) presents a broad emission band in the visible range, with a maximum at $570 \mathrm{~nm}$, implying yellow/green emission, upon excitation with a $330 \mathrm{~nm}$ wavelength, which was determined as the optimum.

\subsection{GO and GQDs in vitro cytotoxicity on heman- gioendothelioma endothelial cells and podocytes}

To compare the cytotoxicity of GO and GQDs, LDH assay was performed on both podocytes and EOMA with several concentrations and different time-points. LD50 was calculated and GQDs resulted less toxic than GO at every time point (Fig. S5 in the ESM).

For EOMA, GO LD50 varied from 32 to $47 \mu \mathrm{g} / \mathrm{mL}$ and for GQDs from 59 to $63 \mu \mathrm{g} / \mathrm{mL}$, between 24 and 72 hours of incubation. LD50 value of podocytes varied from 32 to $35 \mu \mathrm{g} / \mathrm{mL}$ after GO incubation and from 40 to $52 \mu \mathrm{g} / \mathrm{mL}$ after GQDs addition at the same time points. These preliminary data indicated that $30 \mu \mathrm{g} / \mathrm{mL}$ was the more suitable concentration for both permeability assay and cellular uptake experiments.

\subsection{Analysis of GQDs in vitro renal filtration}

To better understand whether GQDs cause a difference in renal clearance or damage the filtration barrier, an in vitro test of glomerular filtration was performed. As described by some of us [22], endothelial cells and podocytes, grown on collagen IV-coated membrane, reflect the physiological resistance to albumin permeability [29]. The BSA permeability was then assessed and the curve reached the plateau after $2 \mathrm{~h}$ reconfirming our previous result. The same assay was subsequently used to establish the time-dependent permeability of GQDs and the trend was compared to BSA curve. It was possible to assess that GQDs, at the concentration of $3 \mathrm{mg} / \mathrm{dL}$, were able to permeate the filtration barrier but showed a lower passage through the barrier compared to BSA (Fig. S6 in the ESM).

\subsection{Evaluation of GQDs cellular uptake}

The different physical and chemical properties of nanomaterials lead to various kinds of interaction with biomolecules and cells [30]. It is therefore important to understand the preferential GQDs cellular uptake pathway on podocytes [27]. The inhibitors concentration (filipin III $1 \mu \mathrm{g} / \mathrm{mL}$, genistein $200 \mu \mathrm{M}$, wortmannin $100 \mathrm{~nm}$, chlorpromazine hydrochloride $10 \mu \mathrm{g} / \mathrm{ml}$, sodium azide $10 \mathrm{mM}$ ), already tested by some of us [27], was reassessed by trypan blue vitality test (Fig. S7(a) in the ESM). The maintenance of the podocyte's cytoskeletal integrity by F-actin immunostaining was monitored after 30 , up to 150 minutes of treatment and no loss of integrity was found after the inhibitor addition compared to the control (Figs. S7(b)-S7(f) in the ESM).

The presence and the distribution of GQDs in podocytes were detected and quantified after 60 minutes. It could be observed that GQDs were taken up by podocytes and retained in both cytoplasm and nucleus. This internalization was partially impaired after the inhibitor's addition. In particular, sodium azide (Fig. 5(b)) partially inhibited cellular uptake as also wortmannin (Fig. 5(c)), whereas chlorpromazine hydrochloride did not inhibit endocytosis of GQDs (Fig. 5(d)). On the contrary absence of GQDs was subsequent to filipin III administration (Fig. 5(e)), as also the marked cytoplasmic reduction following genistein (Fig. 5(f)). The data were confirmed by fluorescence quantification (Fig. 6).

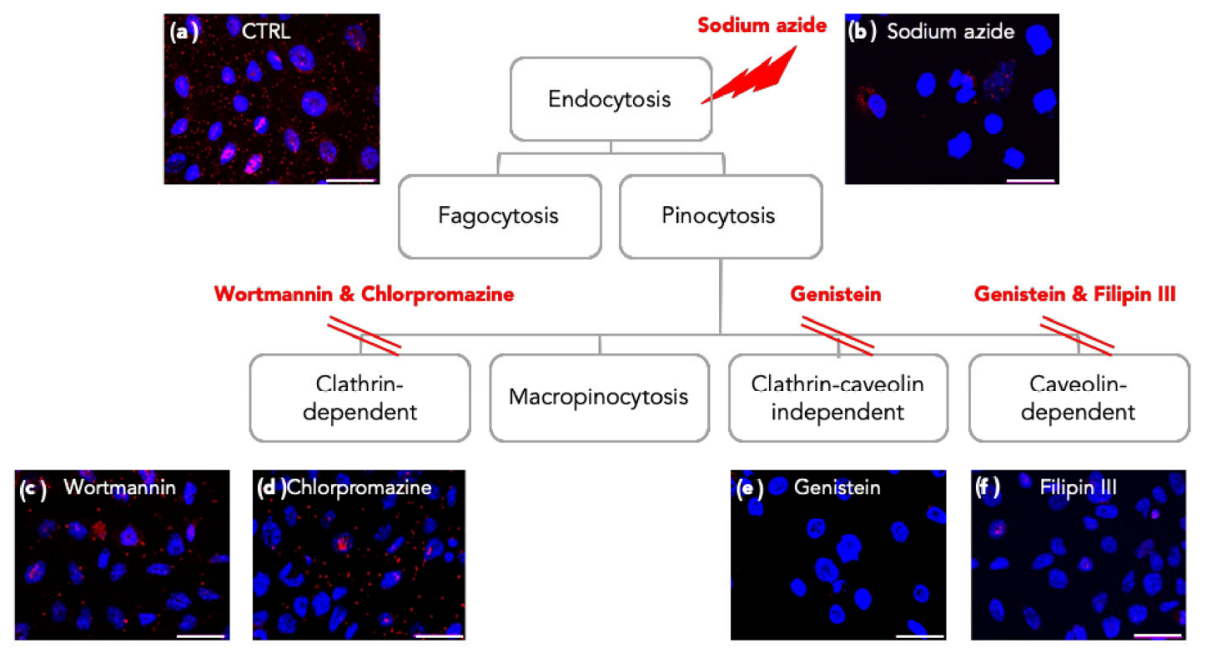

Figure 5 Diagram of uptake internalization. Diagram of GQDs (red) uptake internalization in the podocytes cytoplasm and the nucleus (DAPI in blue) after inhibitor addition compared to (a) the ctrl, $t=60 \mathrm{~min}$; (b) sodium azide; (c) wortmannin; (d) chlorpromazine hydrochloride; (e) genistein; (f) filipin III. Scale bar $50 \mu \mathrm{m}$. 


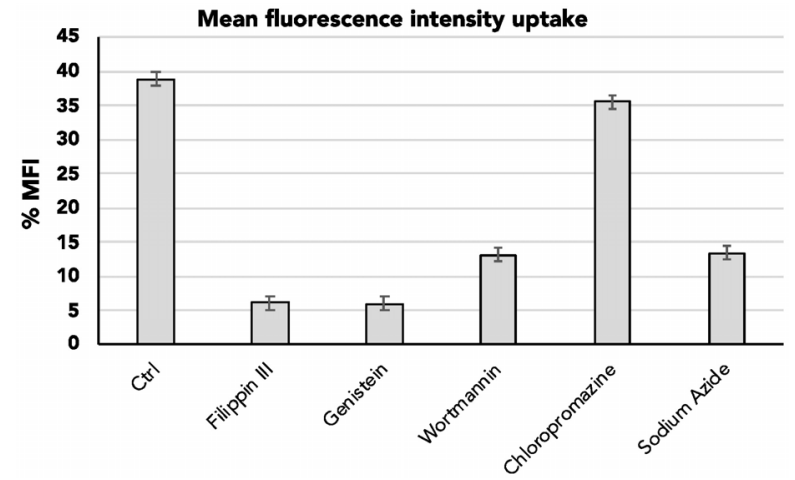

Figure 6 GQDs fluorescence in podocytes. GQDs fluorescence intensity measured in podocytes after the inhibitor addition after 60 minutes.

\subsection{Evaluation of GQDs biodistribution in vivo}

Single-dosing experiments were conducted in mice to assess the in vivo toxicity and biodistribution. Prior fluorescence examination of GQDs distribution, hematoxylin/eosin staining of the organs collected after treatments was performed. First, as expected, no mouse death was observed [31-33], then no clear difference compared to the control animals (Figs. 7(a)-7(e)) was found in their organ structure (Figs. 7(f)-7(j)).

The distribution of GQDs was evaluated in mice kidney, spleen, liver, brain, lung, colon and bladder (Fig. 8). When treated for $24 \mathrm{~h}$ with GQDs 25 and $50 \mu \mathrm{g} / \mathrm{mL}$, no distribution was observed in the kidney, both in the glomerulus and in the tubule (Figs. 8(b) and 8(c)). In liver no GQDs were detected at $25 \mu \mathrm{g} / \mathrm{mL}$, while some NPs were seen after $50 \mu \mathrm{g} / \mathrm{mL}$ treatment (Figs. 8(e) and 8(f)). The compound also accumulated in brain, as reported elsewhere [34] (Figs. 8(h) and 8(i)) and in spleen red pulp at the highest concentration of GQDs (Figs. 8(k) and 8(1)). In lung, bladder, stomach and colon, at both concentrations no GQDs were detected (data not shown) and their presence in urine was evaluated to establish the mice renal clearance (Fig. 9).

At the concentration of $50 \mu \mathrm{g} / \mathrm{mL}$, the amount of GQDs eliminated was less than at the lower concentrations of 25 and $10 \mu \mathrm{g} / \mathrm{mL}$ (Fig. 9).
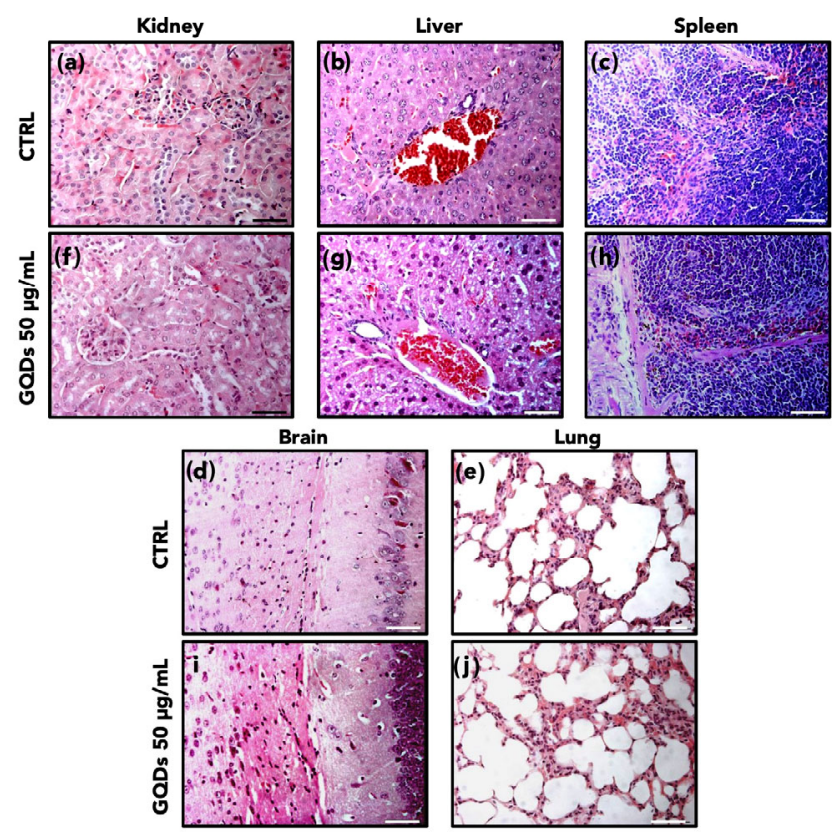

Figure 7 Tissue staining. Hematoxylin/eosin staining of mice tissues after $24 \mathrm{~h}$ GQDs injection. Scale bar $50 \mu \mathrm{m}$.

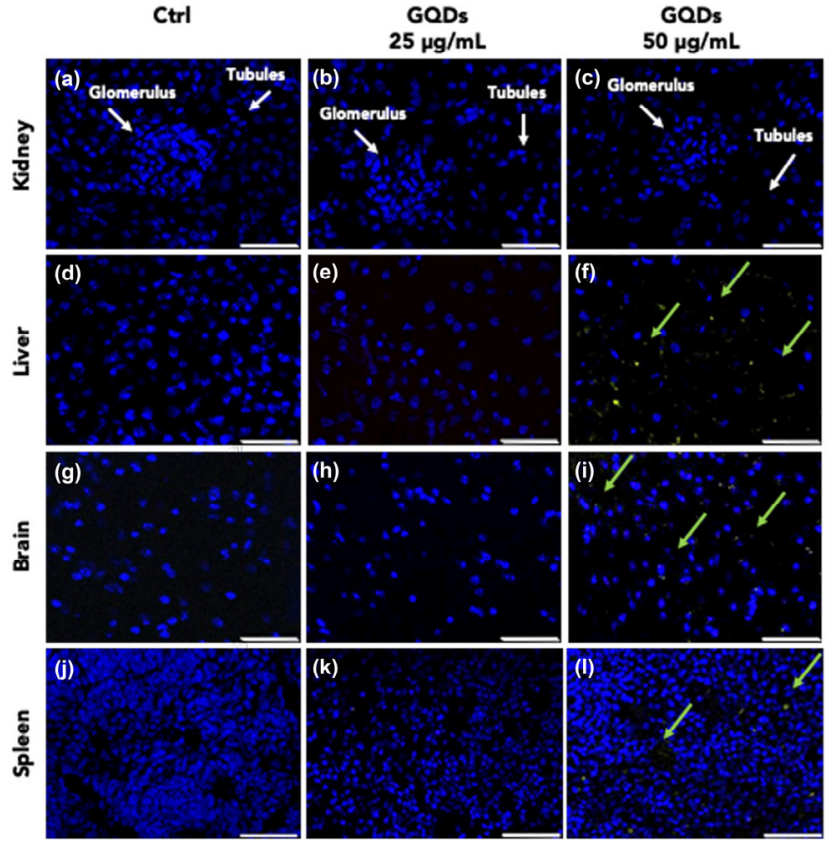

Figure 8 GQDs tissue distribution. GQDs (yellow-green) distribution detected by immunofluorescence in control and treated mouse tissues after 24 hours at $25-50 \mu \mathrm{g} / \mathrm{mL}$. Nuclei DAPI staining (blue). Scale bars $50 \mu \mathrm{m}$.

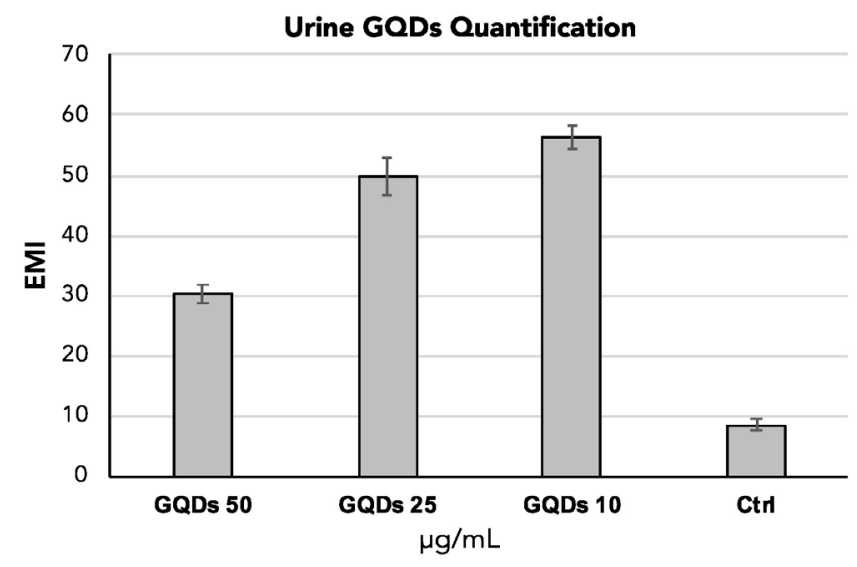

Figure 9 GQDs urine excretion. GQDs quantification in urine of treated (50 and $25 \mu \mathrm{g} / \mathrm{mL}$ ) and control mice by fluorescence.

\section{Discussion}

The reported method to prepare GQDs allowed to obtain tiny nanoparticles with mean diameter of about $3 \mathrm{~nm}$. It is important to note that the yield of GQDs production is often quite low, typically below $10 \%$, and rarely reaching $20 \%$ or more, so there is a major challenge not only in improving the production scale but also in maximizing the synthesis yield and in this case the conversion of GO to GQDs has been virtually $100 \%$. HR-TEM images of an individual particle demonstrate the crystalline structure of the GQDs, where the $0.21 \mathrm{~nm}$ interplanar spacing corresponding to the (100) planes can be observed.

The so prepared material was first studied to determine its cytotoxic profile in comparison to GO on endothelial cells and podocytes, main actors of ultrafiltration barrier, since it represents the more useful prerequisite for renal biosafety. In fact, despite many papers reported about the good biocompatibility of GQDs, the differences of the materials due to the preparation methodology render necessary to explore at first their cytotoxicity. LDH assay and DAPI nuclear staining demonstrated that, in the adopted experimental conditions, 
over $75 \%$ of EOMA, about $65 \%$ of podocytes survive, with nuclei without fragmentation.

These data consequently show a safe profile of GQDs in renal cells, up to the concentration of about $59 \mu \mathrm{g} / \mathrm{mL}$ for EOMA and up to $40 \mu \mathrm{g} / \mathrm{mL}$ for podocytes. These results are perfectly comparable with the studies of GQDs interaction with macrophages and neurons $[34,35]$ but show a greater toxicity than that manifested on MGC-803 gastric cancer cells and on MCF7 breast cancer cells [36]. In vitro studies on Human Adipose-Derived Stem cells (hADSCs) by Kim et al. confirmed the safety of this material with the cell viability higher than $80 \%$ even when the treatment was performed using a $2.0 \mathrm{mg} / \mathrm{mL}$ concentration of GQDs. Considering these results, the researchers used them to tag stem cells and to follow their fate in vivo, proving the possibility to use this material in stem cell therapy imaging [37]. Lower concentrations were used for studies on rat Schwann cells (RSC96) $(10-100 \mu \mathrm{g} / \mathrm{mL}$ ) and also in this case the cell viability was above $90 \%$ [38] and again the GQDs were also used for in vivo imaging, without tissue autofluorescence interference $[36,39]$. Our results are in line also with these reports, taking into consideration not only the difference in the studied materials, but also the differences in the used cell models. Moreover, the herein reported results demonstrated that the safety of GQDs overcomes that of GO, as reported elsewhere [39]. The renal cells that assemble in the glomerulus have the characteristic of forming a special barrier composed of endothelial cells, GBM and podocytes. The in vitro results show that the GQDs kinetics curve, in the passage through this ultrafiltration barrier, is comparable to that of BSA and that the greatest amount of GQDs goes through it in few hours without causing damage. After this time, the quantity of GQDs that cross the membrane remains constant. The GQDs capacity to overcome in vivo the ultrafiltration barrier after $24 \mathrm{~h}$ was confirmed by fluorescence images and quantification, showing that GQDs do not stay in the kidney but pass through it and are released into the urine, showing a fast clearance.

The next step was to deepen into the mechanism by which GQDs pass through the ultrafiltration barrier of podocytes. The use of specific inhibitors is useful to understand the preferential NPs internalization way. Previous studies conducted on cancer cells of different tissue origin described GQDs caveolae-mediated endocytosis in gastric cancer cells and in breast cancer cells, autophagy in lung cancer and in adenocarcinomic human alveolar basal epithelial cells $[36,40]$. Moreover, GQDs have been found to enter by endocytosis and subsequently localized within the lysosome, in neurons, and finally to enter by receptor-mediated endocytosis when conjugated with folic acid [36]. This wide variety of internalization modalities and the absence of studies on renal glomerular cells have necessarily led us to clarify which is the entry way into the podocytes. The investigations, obtained by selectively blocking different entry pathways, support the hypothesis that in these cells the GQDs use, at least in part, an energydependent mechanism of endocytosis, as demonstrated by the weakening of the fluorescence after the addition of sodium azide. After observing the absence of chlorpromazine effect and the relatively few consequences of wortmannin, clathrin-mediated endocytosis can be excluded. In contrast, the decrease in fluorescence intensity following treatment with genistein suggests caveolae-mediated endocytosis or clathrin and caveolae-independent mechanisms. The remarkable decrease of fluorescence after filipin III definitively indicates that caveolae-mediated endocytosis may be the preferential way.

To assess the biosafety of GQDs in vivo single-dosing experiments in mice were performed and the histological absence of morphological changes confirms the good tolerability of GQDs.

As mentioned, not so many in vivo studied have been reported so far. Among the most significant ones, Chong et al. demonstrated that small size GQDs, rich in oxygen, exert very low cytotoxicity and that in mice no accumulation was detected in the principal organs, while a fast excretion through kidney was reported [15]. Other authors took advantage of the intrinsic fluorescence of GQDs to evaluate their biodistribution. In the case of carboxylated carbon dots, obtained by exfoliation of carbon nanofibers, the material fate was followed by fluorescence after injection in the tail vein of $\mathrm{KB}$ tumor bearing mice. The materials tend to accumulate in liver, kidney and tumor tissues without inducing morphological changes as demonstrated by ex vivo analyses [31]. Also post synthesis functionalized polydopamine-coated GQDs were studied in mice and, after tail vein injections, the material was distributed to heart, liver, lung, spleen and kidney and excreted by the latter [41].

In our case, the GQDs distribution and storage in various organs was investigated to understand the post-intravenous administration behavior and the clearance pathways. GQDs presence was detected in liver and in spleen, due to their specific role in blood multiple metabolites trafficking. In addition, their deposition was detected in brain, demonstrating the capacity to cross blood-brain barrier, as already reported by Kim et al. [34]. Moreover, the absence of GQDs in the glomeruli and in the renal tubules suggests the lack of storage and the rapid leakage from the kidney. The renal excretion, reported by Chong and coworkers [15], was studied also for flakes of GO, presenting no more than 3 sheets on height with average lateral dimension lower than $1 \mu \mathrm{m}$, demonstrating that it takes place without changing or damaging the kidneys [42].

\section{Conclusions}

An alternative route for the preparation of GQDs was explored obtaining high yields and the cytotoxicity of the so-obtained materials, presenting an average diameter of $2.9 \mathrm{~nm}$, was studied demonstrating their better behavior with respect to GO. Moreover, in vivo biodistribution experiments were performed, confirming a fast and efficient renal clearance. The GQDs were observed to pass through the glomerular cells without any renal toxicity. The mechanism of internalization was explored and a caveolae-mediated internalization was found in lipid rafts, where ultrafiltration takes place. This allows bypassing lysosomal degradation. However, future multi-dose experiments have to be performed to validate their biocompatibility, assuming that multiple doses should be necessary to obtain pharmacological effects.

Thanks to these evidences, the obtained GQDs result to be really appealing as possible drug-vector to kidney, being a very safe material for this compartment. So, it is possible to envision the use of ultra-small GQDs for drug delivery to ultrafiltration sites, rich in molecules responsible for cell signaling and crucial for cytoskeletal repair, thus participating in the dynamics involved in the treatment of glomerulopathies.

\section{Acknowledgements}

J. M. G.-D. acknowledges Spanish Ministry of Science, Innovation and Universities for his Juan de la Cierva Incorporación research contract (No. IJCI-2016-27789). This project has received funding from the European Union's Horizon 2020 Research and Innovation Programme under the Marie 
Skłodowska-Curie Grant Agreement No. 734834 (INFUSION) and No. 734381 (CARBO-IMmap), and from MIUR. ICN2 is supported by the Severo Ochoa program from Spanish MINECO (No. SEV-2017-0706).

Funding note : Open Access funding provided by Università degli Studi di Trieste within the CRUICARE Agreement.

Electronic Supplementary Material: Supplementary material (six figures) is available in the online version of this article at https://doi.org/10.1007/s12274-020-3096-y.

Open Access This article is licensed under a Creative Commons Attribution 4.0 International License, which permits use, sharing, adaptation, distribution and reproduction in any medium or format, as long as you give appropriate credit to the original author(s) and the source, provide a link to the Creative Commons licence, and indicate if changes were made.

The images or other third party material in this article are included in the article's Creative Commons licence, unless indicated otherwise in a credit line to the material. If material is not included in the article's Creative Commons licence and your intended use is not permitted by statutory regulation or exceeds the permitted use, you will need to obtain permission directly from the copyright holder.

To view a copy of this licence, visit http://creativecommons.org/licenses/by/4.0/.

\section{References}

[1] Novoselov, K. S.; Geim, A. K.; Morozov, S. V.; Jiang, D.; Zhang, Y.; Dubonos, S. V.; Grigorieva, I. V.; Firsov, A. A. Electric field effect in atomically thin carbon films. Science 2004, 306, 666-669.

[2] Chen, D.; Feng, H. B.; Li, J. H. Graphene oxide: Preparation, functionalization, and electrochemical applications. Chem. Rev. 2012, 112, 6027-6053.

[3] Reina, G.; González-Domínguez, J. M.; Criado, A.; Vázquez, E.; Prato, M.; Bianco, A. Promises, facts and challenges for graphene in biomedical applications. Chem. Soc. Rev. 2017, 46, 4400-4416.

[4] Sun, X. M.; Liu, Z.; Welsher, K.; Robinson, J. T.; Goodwin, A.; Zaric, S.; Dai, H. J. Nano-graphene oxide for cellular imaging and drug delivery. Nano Res. 2008, 1, 203-212.

[5] Georgakilas, V.; Perman, J. A.; Tucek, J.; Zboril, R. Broad family of carbon nanoallotropes: Classification, chemistry, and applications of fullerenes, carbon dots, nanotubes, graphene, nanodiamonds, and combined superstructures. Chem. Rev. 2015, 115, 4744-4822.

[6] Wick, P.; Louw-Gaume, A. E.; Kucki, M.; Krug, H. F.; Kostarelos, K.; Fadeel, B.; Dawson, K.; Salvati, A.; Vázquez, E.; Ballerini, L. et al. Classification framework for graphene-based materials. Angew. Chem., Int. Ed. 2014, 53, 7714-7718.

[7] Zhou, S. H.; Xu, H. B.; Gan, W.; Yuan, Q. H. Graphene quantum dots: Recent progress in preparation and fluorescence sensing applications. RSC Adv. 2016, 6, 110775-110788.

[8] Das, P.; Ganguly, S.; Banerjee, S.; Das, N. C. Graphene based emergent nanolights: A short review on the synthesis, properties and application. Res. Chem. Intermed. 2019, 45, 3823-3853.

[9] Peng, J.; Gao, W.; Gupta, B. K.; Liu, Z.; Romero-Aburto, R.; Ge, L. H.; Song, L.; Alemany, L. B.; Zhan, X. B.; Gao, G. H. et al. Graphene quantum dots derived from carbon fibers. Nano Lett. 2012, 12, 844-849.

[10] Blanco, E.; Blanco, G.; Gonzalez-Leal, J. M.; Barrera, M. C.; Domínguez, M.; Ramirez-del-Solar, M. Green and fast synthesis of amino-functionalized graphene quantum dots with deep blue photoluminescence. J. Nanopart. Res. 2015, 17, 214.

[11] Xu, H. B.; Zhou, S. H.; Xiao, L. L.; Yuan, Q. H.; Gan, W. Timeefficient syntheses of nitrogen and sulfur co-doped graphene quantum dots with tunable luminescence and their sensing applications. RSC Adv. 2016, 6, 36554-36560.

[12] Li, X. M.; Rui, M. C.; Song, J. Z.; Shen, Z. H.; Zeng, H. B. Carbon and graphene quantum dots for optoelectronic and energy devices: A review. Adv. Funct. Mater. 2015, 25, 4929-4947.

[13] Wang, Y.; Li, Z. H.; Li, J. H.; Lin, Y. H. Graphene and graphene oxide: Biofunctionalization and applications in biotechnology. Trends Biotechnol. 2011, 29, 205-212.

[14] Wang, S. J.; Cole, I. S.; Li, Q. The toxicity of graphene quantum dots. RSC Adv. 2016, 6, 89867-89878.

[15] Chong, Y.; Ma, Y. F.; Shen, H.; Tu, X. L.; Zhou, X.; Xu, J. Y.; Dai, J. W.; Fan, S. J.; Zhang, Z. J. The in vitro and in vivo toxicity of graphene quantum dots. Biomaterials 2014, 35, 5041-5048.

[16] de Menezes, F. D.; dos Reis, S. R. R.; Pinto, S. R.; Portilho, F. L.; Do Vale Chaves e Mello, F.; Helal-Neto, E.; da Silva de Barros, A. O.; Rebêlo Alencar, L. M.; de Menezes, A. S.; dos Santos, C. C. et al. Graphene quantum dots unraveling: Green synthesis, characterization, radiolabeling with $99 \mathrm{mTc}$, in vivo behavior and mutagenicity. Mater. Sci. Eng. C 2019, 102, 405-414.

[17] Roy, P.; Periasamy, A. P.; Lin, C. Y.; Her, G. M.; Chiu, W. J.; Li, C. L.; Shu, C. L.; Huang, C. C.; Liang, C. T.; Chang, H. T. Photoluminescent graphene quantum dots for in vivo imaging of apoptotic cells. Nanoscale 2015, 7, 2504-2510.

[18] Zhang, J. H.; Sun, T.; Niu, A. P.; Tang, Y. M.; Deng, S.; Luo, W.; Xu, Q.; Wei, D. P.; Pei, D. S. Perturbation effect of reduced graphene oxide quantum dots (rGOQDs) on aryl hydrocarbon receptor (AhR) pathway in zebrafish. Biomaterials 2017, 133, 49-59.

[19] Wang, Z. G.; Zhou, R.; Jiang, D.; Song, J. E.; Xu, Q.; Si, J.; Chen, Y. P.; Zhou, X.; Gan, L.; Li, J. Z. et al. Toxicity of graphene quantum dots in zebrafish embryo. Biomed. Environ. Sci. 2015, 28, 341-351.

[20] Song, H.; Wang, Y. H.; Wang, J.; Wang, G. Q.; He, J. H.; Wei, H. Y.; Luo, S. Z. Preparation and biodistribution of ${ }^{131}$ I-labeled graphene quantum dots. J. Radioanal. Nuclear Chem. 2018, 316, 685-690.

[21] Greka, A.; Mundel, P. Cell biology and pathology of podocytes. Annu. Rev. Physiol. 2012, 74, 299-323.

[22] Li, M.; Corbelli, A.; Watanabe, S.; Armelloni, S.; Ikehata, M.; Parazzi, V.; Pignatari, C.; Giardino, L.; Mattinzoli, D.; Lazzari, L. et al. Three-dimensional podocyte-endothelial cell co-cultures: Assembly, validation, and application to drug testing and intercellular signaling studies. Eur. J. Pharm. Sci. 2016, 86, 1-12.

[23] Benzing, T. Signaling at the slit diaphragm. J. Am. Soc. Nephrol. 2004, 15, 1382-1391.

[24] Drenckhahn, D.; Franke, R. P. Ultrastructural organization of contractile and cytoskeletal proteins in glomerular podocytes of chicken, rat, and man. Lab. Invest. 1988, 59, 673-682.

[25] Pavenstadt, H.; Kriz, W.; Kretzler, M. Cell biology of the glomerular podocyte. Physiol. Rev. 2003, 83, 253-307.

[26] Ruggiero, A.; Villa, C. H.; Bander, E.; Rey, D. A.; Bergkvist, M.; Batt, C. A.; Manova-Todorova, K.; Deen, W. M.; Scheinberg, D. A.; McDevitt, M. R. Paradoxical glomerular filtration of carbon nanotubes. Proc. Natl. Acad. Sci. USA 2010, 107, 12369-12374.

[27] Colombo, C.; Li, M.; Watanabe, S.; Messa, P.; Edefonti, A.; Montini, G.; Moscatelli, D.; Rastaldi, M. P.; Cellesi, F. Polymer nanoparticle engineering for podocyte repair: From in vitro models to new nanotherapeutics in kidney diseases. ACS Omega 2017, 2, 599-610.

[28] Hummers, W. S. Jr.; Offeman, R. E. Preparation of graphitic oxide. J. Am. Chem. Soc. 1958, 80, 1339.

[29] Lindström, K. E.; Johnsson, E.; Haraldsson, B. Glomerular charge selectivity for proteins larger than serum albumin as revealed by lactate dehydrogenase isoforms. Acta Physiol. Scand. 1998, 162, 481-488.

[30] Rejman, J.; Oberle, V.; Zuhorn, I. S.; Hoekstra, D. Size-dependent internalization of particles via the pathways of clathrin- and caveolae-mediated endocytosis. Biochem. J. 2004, 377, 159-169.

[31] Nurunnabi, N.; Khatun, Z.; Huh, K. M.; Park, S. Y.; Lee, D. Y.; Cho, K. J.; Lee, Y. K. In vivo biodistribution and toxicology of carboxylated graphene quantum dots. ACS Nano 2013, 7, 6858-6867.

[32] Huang, X. L.; Zhang, F.; Zhu, L.; Choi, K. Y.; Guo, N.; Guo, J. K.; Tackett, P.; Anilkumar, G.; Liu, Q.; Quan, H. S. et al. Effect of injection routes on the biodistribution, clearance, and tumor uptake of carbon dots. ACS Nano 2013, 7, 5684-5693.

[33] Tao, H. Q.; Yang, K.; Ma, Z.; Wan, J. M.; Zhang, Y. J.; Kang, Z. H.; Liu, Z. In vivo NIR fluorescence imaging, biodistribution, and toxicology of photoluminescent carbon dots produced from carbon nanotubes and graphite. Small 2012, 8, 281-290. 
[34] Kim, D.; Yoo, J. M.; Hwang, H.; Lee, J.; Lee, S. H.; Yun, S. P.; Park, M. J.; Lee, M.; Choi, S.; Kwon, S. H. et al. Graphene quantum dots prevent $\alpha$-synucleinopathy in Parkinson's disease. Nat. Nanotechnol. 2018, 13, 812-818.

[35] Qin, Y.; Zhou, Z. W.; Pan, S. T.; He, Z. X.; Zhang, X.; Qiu, J. X.; Duan, W.; Yang, T. X.; Zhou, S. F. Graphene quantum dots induce apoptosis, autophagy, and inflammatory response via p38 mitogenactivated protein kinase and nuclear factor- $\mathrm{\kappa B}$ mediated signaling pathways in activated THP-1 macrophages. Toxicology 2015, 327, $62-76$.

[36] Wu, C. Y.; Wang, C.; Han, T.; Zhou, X. J.; Guo, S. W.; Zhang, J. Y. Insight into the cellular internalization and cytotoxicity of graphene quantum dots. Adv. Healthc. Mater. 2013, 2, 1613-1619.

[37] Kim, J.; Song, S. H.; Jin, Y.; Park, H. J.; Yoon, H.; Jeon, S.; Cho, S. W. Multiphoton luminescent graphene quantum dots for in vivo tracking of human adipose-derived stem cells. Nanoscale 2016, 8 , 8512-8519.

[38] Zhu, S. J.; Zhou, N.; Hao, Z. Y.; Maharjan, S.; Zhao, X. H.; Song, Y. B.; Sun, B.; Zhang, K.; Zhang, J. H.; Sun, H. C. et al.
Photoluminescent graphene quantum dots for in vitro and in vivo bioimaging using long wavelength emission. RSC $A d v$. 2015, 5, 39399-39403.

[39] Wang, X. J.; Sun, X.; Lao, J.; He, H.; Cheng, T. T.; Wang, M. Q.; Wang, S. J.; Huang, F. Multifunctional graphene quantum dots for simultaneous targeted cellular imaging and drug delivery. Colloid Surf. B 2014, 122, 638-644.

[40] Xie, Y. C.; Wan, B.; Yang, Y.; Cui, X. J.; Xin, Y.; Guo, L. H. Cytotoxicity and autophagy induction by graphene quantum dots with different functional groups. J. Environ. Sci. 2019, 77, 198-209.

[41] Nurunnabi, N.; Khatun, Z.; Nafiujjaman, M.; Lee, D. Y.; Lee, Y. Surface coating of graphene quantum dots using mussel-inspired polydopamine for biomedical optical imaging. ACS Appl. Mater. Interfaces 2013, 5, 8246-8253.

[42] Jasim, D. A.; Murphy, S.; Newman, L.; Mironov, A.; Prestat, E.; McCaffrey, J.; Ménard-Moyon, C.; Rodrigues, A. F.; Bianco, A.; Haigh, S. et al. The effects of extensive glomerular filtration of thin graphene oxide sheets on kidney physiology. ACS Nano 2016, 10, 10753-10767. 